Urologe 2022 $61: 41-51$

https://doi.org/10.1007/s00120-021-01621-6

Angenommen: 29. Juni 2021

Online publiziert: 23. August 2021

๑) Springer Medizin Verlag GmbH, ein Teil von Springer Nature 2021
Die Autoren Tim Schiereck, Sarah Yeldan und Dimitri Barski haben zu gleichen Teilen zum Manuskript beigetragen. Die Arbeit erfolgte mit der Unterstützung von GeSRU Academics (Mitglieder der Forschungsgruppen Infektiologie und funktionelle Urologie: Jennifer Kranz, Laila Schneidewind und Dimitri Barski)

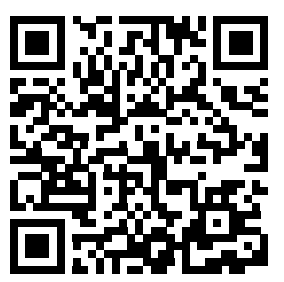

QR-Code scannen \& Beitrag online lesen

\title{
Mikrobiomanalyse der Harnblase und probiotische Therapieoptionen bei Frauen mit rezidivierenden Harnwegsinfektionen
}

T. Schiereck ${ }^{1} \cdot$ S. Yeldan ${ }^{1} \cdot$ J. Kranz Kr, $^{2}$ L. Schneidewind ${ }^{4} \cdot$ F. Wagenlehner ${ }^{5} \cdot$ I. Wieters $^{6}$. M. J. G. T. Vehreschild ${ }^{6}$. T. Otto ${ }^{1,7} \cdot$ D. Barski

${ }^{1}$ Urologische Klinik, Lukaskrankenhaus Neuss, Rheinland Klinikum, Neuss, Deutschland; ${ }^{2}$ Klinik für Urologie und Kinderurologie, St.-Antonius-Hospital Eschweiler, Akademisches Lehrkrankenhaus der RWTH Aachen, Eschweiler, Deutschland; ${ }^{3}$ Universitätsklinik und Poliklinik für Urologie, Universitätsklinikum Halle (Saale), Halle (Saale), Deutschland; ${ }^{4}$ Urologische Klinik und Poliklinik, Universitätsmedizin Rostock, Rostock, Deutschland; ${ }^{5}$ Klinik für Urologie, Kinderurologie und Andrologie, Universitätsklinikum Gießen, Gießen, Deutschland; ${ }^{6}$ Zentrum für Innere Medizin, Infektiologie, Universitätsklinikum Frankfurt, Goethe Universität Frankfurt, Frankfurt am Main, Deutschland; ' ${ }^{7}$ Universität Duisburg-Essen, Essen, Deutschland

Zusammenfassung

Um die Frequenz rezidivierender Harnwegsinfektionen ( $\mathrm{rHWl}$ ) und die damit verbundene fortwährende Zunahme multiresistenter Uropathogene zu reduzieren, werden neuartige Präventionsmaßnahmen und Therapieansätze benötigt. Ziel dieser Übersichtsarbeit ist es, die aktuell verfügbare Evidenz über das Mikrobiom der Harnblase gesunder Frauen und solcher mit rHWI systematisch zu präsentieren. Außerdem werden relevante Studien zur Wirksamkeit von Probiotika bei rHWI strukturiert dargestellt. Hierdurch soll der gegenwärtige Stand der Forschung und ein Ausblick auf eine Therapie abseits der üblichen antimikrobiellen Behandlungsoptionen gegeben werden.

\section{Schlüsselwörter}

Weibliche Harnblase · Multiresistente Erreger · 16S-rRNA-Sequenzierung · Lactobacillus · Fäkaler Mikrobiotatransfer

Rezidivierende Harnwegsinfektionen (rHWI) treten definitionsgemäß mindestens 2-mal binnen 6 Monaten oder 3-mal binnen 12 Monaten auf [8]. Die Inzidenz liegt bei 1-5\%. Neben körperlichen (z.B. Schmerz) und psychischen Belastungen (z. B. Stress, Angst) treten auch eine eingeschränkte Lebensqualität, sowie sozioökonomische Faktoren (z.B. eingeschränkte Arbeitsfähigkeit und Sozialkontakte) auf. Zudem ist vor allem die mit der wiederholten Exposition gegenüber Antibiotika assoziierte Zunahme von bakteriellen Resistenzen von großer Bedeutung [11, 48].

\section{Einleitung}

Der früher postulierte Schwellenwert einer signifikanten Bakteriurie von $10^{5}$ Erregern pro $\mathrm{ml} \mathrm{im} \mathrm{Mittelstrahlurin} \mathrm{(Kass-Zahl)}$ rückt zunehmend in den Hintergrund. Wichtiger ist die klinische Symptomatik, was unterschiedliche Leitlinien aufgreifen $[3,8]$. Ein möglicher Grund für negative Urinkulturen bei symptomatischen Patienten ist das Vorliegen von atypischen oder anaeroben Bakterien, oder Bakterien unterschiedlicher Taxa (polymikrobielle Infektion; [9, 31]). Bei rezidivierenden Harnwegsinfektionen (rHWI) sollten komplizierende Faktoren wie beispielsweise funktionelle und/oder anatomische Veränderungen unbedingt ausgeschlos- 


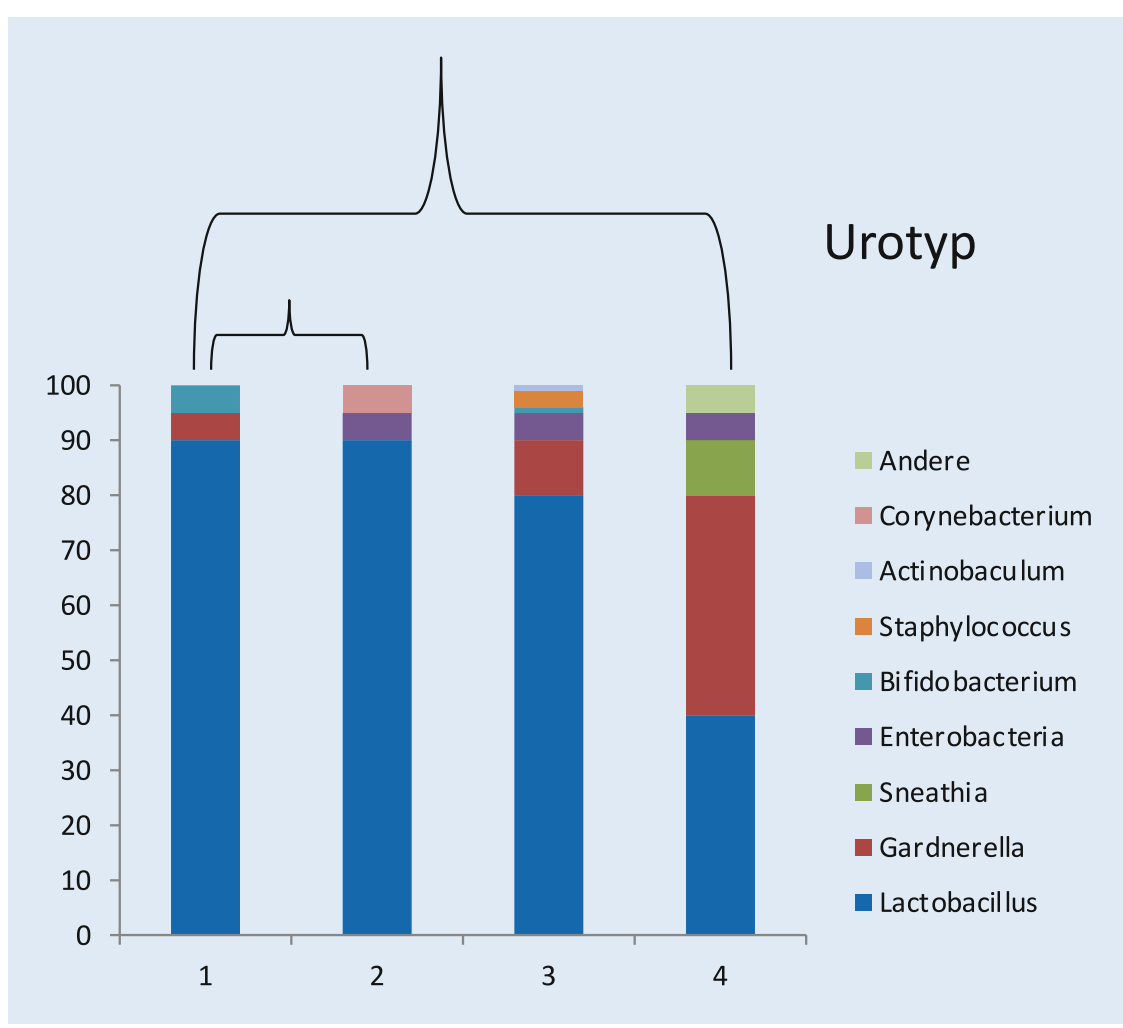

Abb. $1 \Delta$ Es werden beispielhaft vier 16S-rRNA-Squenzierungen mit Histogramm (unten) und Dendogramm (oben) dargestellt. Das Histogramm zeigt die bakteriellen Taxa, die in jeder Probe erkannt wurden, als Prozentsatz der gesamten klassifizierten Sequenz-Reads an. Jede Farbe steht je nach Definition für eine andere Familie oder Gattung. Durch den Vergleich des Dendrogramm-Clusterings mit der Klassifizierung können Urotypen definiert und auf der Grundlage des dominanten (häufigsten) klassifizierten Organismus benannt werden. (In Anlehnung an Brubaker [9])

sen werden. Die aktuelle S3-Leitlinie der Arbeitsgemeinschaft der Wissenschaftlichen Medizinischen Fachgesellschaften e.V. (AWMF) zu unkomplizierten Harnwegsinfektionen $(\mathrm{HWI})$ bei erwachsenen Patienten empfiehlt bei prämenopausalen, nicht schwangeren Frauen zunächst die Durchführung einer Urinkultur sowie Sonographie des Harntraktes inklusive Restharnbestimmung [3].

Zur exakten Identifizierung und Differenzierung von Uropathogenen können heute moderne Methoden wie die 16S-rRNA-Sequenzierung und „expanded quantitative urine culture" (EQUC) eingesetzt werden [18]. Die EQUC nutzt dabei optimierte erweiterte Protokolle mit verschiedenen Urinmengen, Kombinationen von Nährböden, Agarmedien und Inkubationszeiten [32]. Price et al. empfehlen ein Stufensystem, um bei symptomatischen Patientinnen ohne Erregernachweis in der Kultur weitere Pathogene mittels erweiterter EQUC zu detektieren [32]. Die 16S-rRNA-Squenzierung verwendet kenversicherung erfolgt. Aufgrund der Einschränkungen dieser Technologie, einschließlich der Interpretierbarkeit und der fehlenden Möglichkeit, Informationen über bakterielle Antibiotikaresistenzen abzurufen, ist es wichtig, auch die zusätzlichen Kosten für wachstumsbasierte Empfindlichkeitstests zu berücksichtigen. Der Preis einer Urinkultur beträgt etwa $40 €$, und erhöht sich auf etwa $330 €$ bei zusätzlichen PCR (,polymerase chain reaction") und Tests auf Pilz- oder anaerobe Spezies [29].

Die Existenz eines Harnblasenmikrobioms ist ein momentan kontrovers diskutiertes, hoch aktuelles Thema und Gegenstand der Forschung. Ein entscheidender Einfluss auf Genese und Verlauf chronischer Erkrankungen wie beispielsweise die interstitielle Zystitis wird angenommen [40]. Die Vorstellung von sterilem Urin scheint daher überworfen [18]. D Abb. 2 zeigt eine Übersicht über das weibliche Kernmikrobiom des Urogenitalsystems und dessen Abweichungen bei HWI. Die möglichen Funktionen eines intakten Blasenmikrobioms bestehen in einer protektiven Barriere gegenüber pathogenen Erreger, indem beispielsweise deren Adhäsion verhindert wird, der Produktion antimikrobieller Wirkstoffe und Aktivierung der körpereigenen Immunabwehr [47]. Mehrere aktuelle Studien konnten das Vorhandensein einer natürlichen mikrobiellen Besiedelung der Harnblase nachweisen.

Alternativen zur antibiotischen Therapie rücken zukünftig weiter in den Vordergrund [4, 28, 31].

Gemäß der AWMF-S3-Leitlinie zu unkomplizierten HWI folgen die Präventionsmaßnahmen bei Frauen mit rHWI einem Stufenkonzept. Vor Einleiten einer antimikrobiellen Langzeitbehandlung sollte ausführlich über die Vermeidung von Risiken (z.B. geringe Trinkmenge, Unterkühlung, übertriebene Intimhygiene) beraten werden (Evidenzgrad Ib, Empfehlungsgrad A). Eine Immunprophylaxe durch orale Gabe eines Escherichia-coli-Lysats (OM-89) über 3 Monate (Evidenzgrad la, Empfehlungsgrad B) oder durch drei parenterale Injektionen von inaktivierten spezifizierten Enterobakterien im Abstand von einer Woche kann erwogen werden (Evidenzgrad Ib, Empfehlungsgrad C). Auch Man- 


$\begin{array}{ll}\begin{array}{l}\text { Mikrobiom } \\ \text { vorherrschend } \\ \text { bei der Frau }\end{array} & \begin{array}{l}\text { Prevotella } \\ \text { Sneathia }\end{array} \\ \begin{array}{l}\text { Vorherrschendes } \\ \text { Mikrobiom der } \\ \text { Harnblase }\end{array} & \begin{array}{l}\text { Lactobacillus } \\ \text { Streptococcus } \\ \text { Corynebacterium } \\ \text { Ureaplasma } \\ \text { Staphylococcus }\end{array} \\ \begin{array}{l}\text { Auslöser von } \\ \text { Harnwegsinfektionen }\end{array} & \begin{array}{l}\text { Escherichia coli } \\ \text { Proteus mirabilis } \\ \text { Enterococcus }\end{array}\end{array}$

nose kann in Betracht gezogen werden (Evidenzgrad Ib, Empfehlungsgrad C; [3]). Die Leitlinie der "European Association of Urology" (EAU) begrenzt den Einsatz von D-Mannose derzeit auf klinische Studien [8]. Eine Alternative zu den oben genannten Maßnahmen könnten auch Probiotika wie z.B. Lactobacilli darstellen [24, 38]. Probiotika sind Mikroorganismen, die in therapeutischer Dosierung in verschiedenen Applikationsformen verabreicht werden können. Hierdurch soll die natürliche Barrierefunktion der Vaginalschleimhaut aufrechterhalten oder wiederaufgebaut werden, um die Migration pathogener Erreger zu verhindern [37]. Ein protektiver Effekt von Lactobacilli auf die Entwicklung von symptomatischen HWI konnte bisher jedoch nicht eindeutig nachgewiesen werden [38]. Gegenwärtig gibt es keine Evidenz zum Einsatz von Probiotika in dieser Indikation [8]. Bei hohem Leidensdruck der Patientin sollte erst nach Versagen der nicht antimikrobiellen Prävention eine kontinuierliche Langzeitantibiotikaprophylaxe über 3-6 Monate (Evidenzgrad IV, Empfehlungsgrad B) oder ggf. eine postkoitale Prophylaxe mit Verabreichung einer Einzeldosis eines Antibiotikums erfolgen (Evidenzlevel lb, Empfehlungsgrad B). Nach der EAU-Leitlinie sind sowohl eine kontinuierliche niedrig dosierte als auch eine postkoitale antimikrobielle Prophylaxe geeignet, die Rate rHWI zu reduzieren (Evidenzgrad 1b, starke Empfehlung; [8]). Weitere Forschungsvorhaben sind wünschenswert, um die Rolle der nicht-antibiotischen Therapie und Prävention von rHWI zu klären.

Ziel dieser Übersichtsarbeit ist es, aktuell verfügbare Evidenz über die Bedeutung
Gardnerella

Bifidobacterium

Actinobaculum

Actinomyces

Aerococcus

von oral und vaginal applizierten Probiotika wurden aufgrund von fehlenden Sequenzierungsuntersuchungen auch Publikationen mit Analysen von herkömmlichen Urinkulturen eingeschlossen. Sowohl die Dauer der Therapie als auch die Nachsorge wurden erhoben.

\section{Ergebnisse}

Abb. $2 \triangleleft$ Übersicht über das Mikrobiom der Harnblase in Anlehnung zu [10] der Blasenmikrobiome gesunder Frauen und solcher mit rHWI systematisch zu präsentieren. Außerdem werden relevante Studien zur Wirksamkeit von Probiotika bei rHWI strukturiert dargestellt. Hierdurch soll der gegenwärtige Stand der Forschung und ein Ausblick auf eine Therapie abseits der üblichen antimikrobiellen Behandlungsoptionen gegeben werden.

\section{Methodik}

Für diese narrative Übersichtsarbeit wurde eine selektive Literaturrecherche über MEDLINE für den Zeitraum vom Januar 1990 bis Dezember 2020 durchgeführt. Es wurden Volltextpublikationen in deutscher und englischer Sprache berücksichtigt. Es wurden nur randomisierte klinische Studien (RCT) und klinische Studien inkludiert, die sich auf die mikrobiologische Urinanalyse von Frauen mit und ohne rHWI fokussierten. Zudem schlossen wir kleinere retrospektive Studien zur Therapie mit Probiotika oder anderen mikrobiotabasierten Therapien ein. Ausgeschlossen wurden Studien mit ausschließlich männlichen Teilnehmern und/oder Fokus auf sexuell übertragbare Erkrankungen. Bezüglich der Mikrobiotabestimmung aus dem Urin gesunder Frauen schlossen wir nur jeneStudien ein, welche eine 16S-rRNA-Sequenzierung für die Speziesbestimmung nutzten, um so ein genaueres Bild des "gesunden" Blasenmikrobioms zu erhalten. Für Studien bei Frauen mit rHWI wurden zusätzlich zur Sequenzierung auch EQUC-Untersuchungen evaluiert. Die Art der Uringewinnung (Mittelstrahl- oder Katheterurin oder suprapubische Punktion) ist hierbei differenziert angegeben. Bei Studien zum Einsatz
Insgesamt wurden 35 Studien identifiziert und in die finale Auswertung eingeschlossen (s. - Tab. 1, 2, 3 und 4). Auffällig ist eine große Heterogenität in Bezug auf die Methodik und Studienpopulation. Insbesondere Studien mit großer Teilnehmerzahl fehlen. Die überwiegende Studienanzahl untersucht das Blasenmikrobiom gesunder Frauen, die keine typischen Symptome einer HWI aufwiesen ( $n=12)$. Dagegen existieren bis dato nur wenige Studien, die das Mikrobiom von Frauen mit rHWI untersuchen.

\section{Blasenmikrobiomanalyse gesunder Frauen}

Die mit Hilfe von 16S-rRNA-Sequenzierung durchgeführten Untersuchungen der eingeschlossenen Studien haben gezeigt, dass das Blasenmikrobiom gesunder Frauen v.a. eine breite natürliche Diversität aufweist. Vorherrschend waren die Gattungen Lactobacillus spp., Prevotella spp. und Gardnerella sowie Corynebacterium spp., Streptococcus spp., Actinomyces spp., Staphylococcus spp. und die Phyla Firmicutes, Bacteroidetes, Actinobacteria, Proteobacteria und Fusobacteria (s. - Tab. 1). Eine alters- und geschlechtsabhängige Untersuchung wurde bei Lewis et al. durchgeführt, in welcher $>50 \%$ der Proben beider Geschlechter von Bakterien des Phylums Firmicutes dominiert wurden. Die Mikrobiomdiversität der Frauen war heterogener mit einem vermehrten Vorkommen von Actinobacteria und Bacteroidetes-Phyla [27]. Price et al. haben das Mikrobiom aus Mittelstrahlurin und periurethralem Abstrich von 8 asymptomatischen prämenopausalen Frauen über einen Zeitraum von 3 Monaten untersucht. Eine Veränderung der Mikrobiotavariabilität in Abhängigkeit vom Zeitpunkt des Menstruationszyklus, sowie eine Abhängigkeit von der sexuellen Aktivität wurde 


\begin{tabular}{|c|c|c|c|c|c|c|c|c|}
\hline 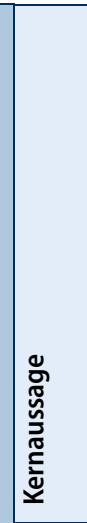 & 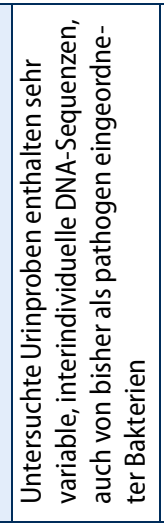 & 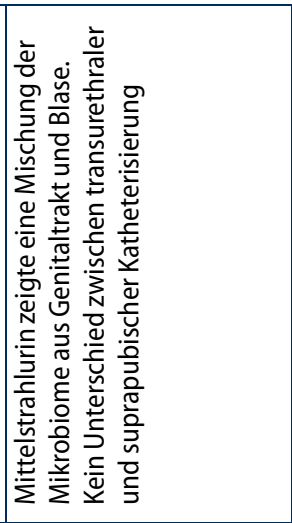 & 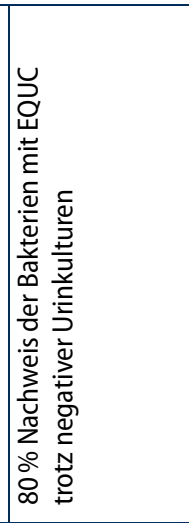 &  & 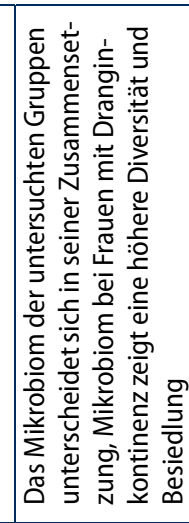 & 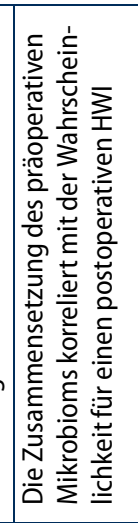 &  & 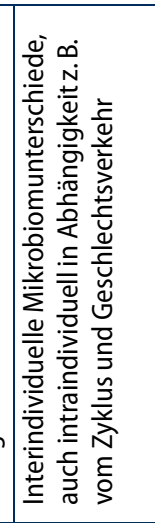 \\
\hline 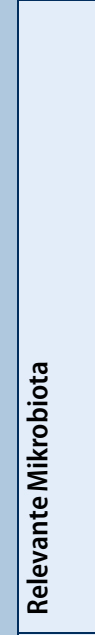 & 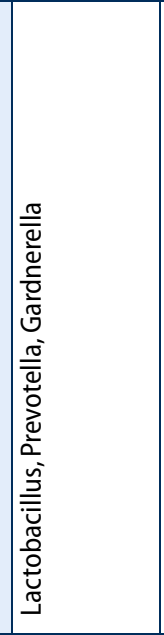 &  &  & 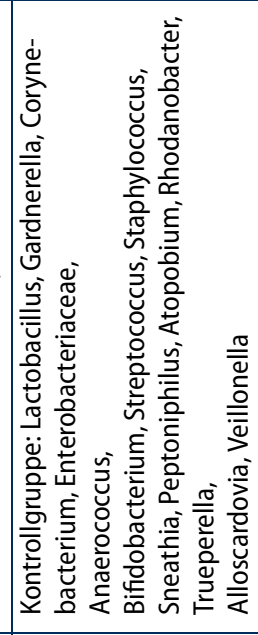 & 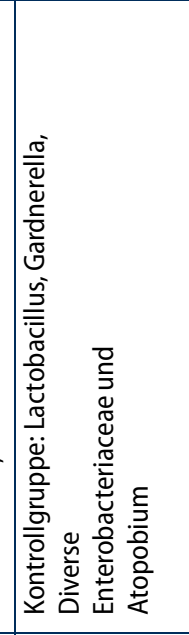 & 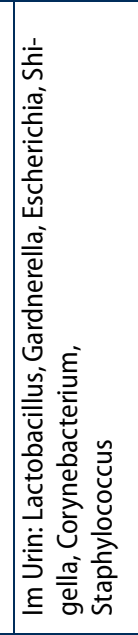 & 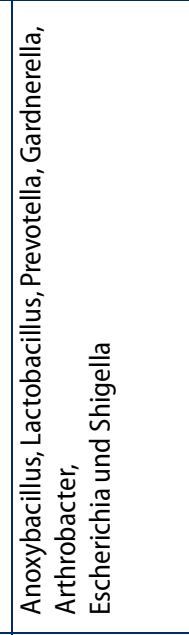 & 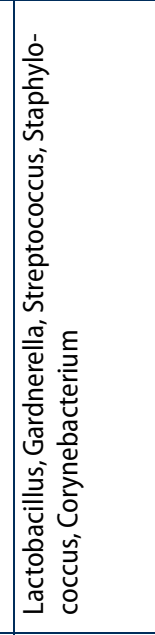 \\
\hline 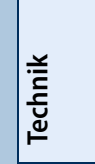 & 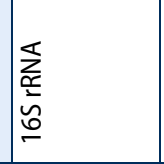 &  & 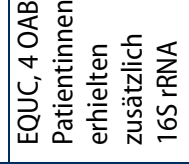 & 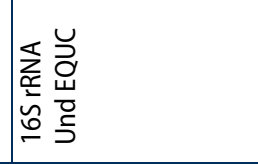 & 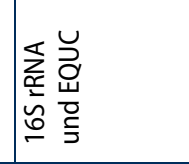 & 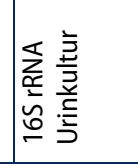 & 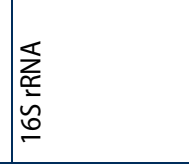 & 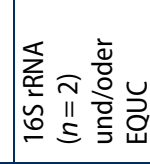 \\
\hline 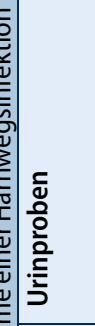 & 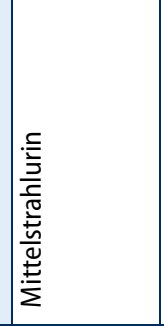 &  & 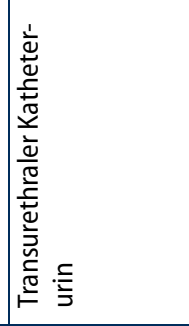 & 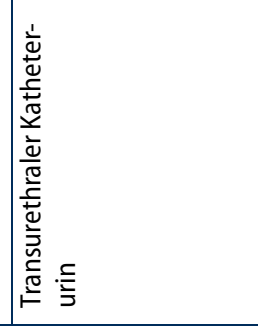 & 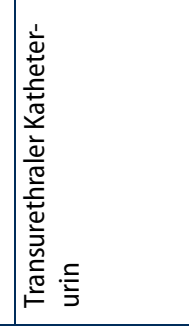 & 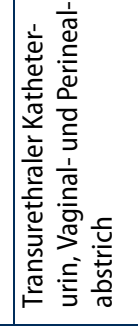 & 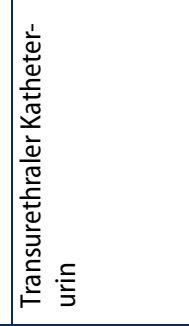 & 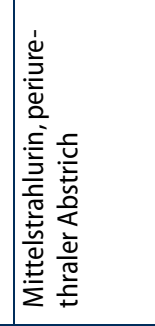 \\
\hline  & 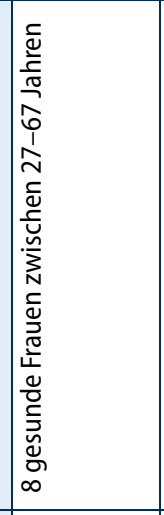 & 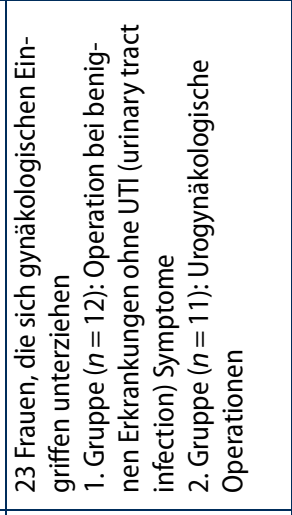 & 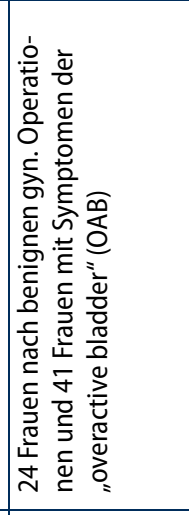 & 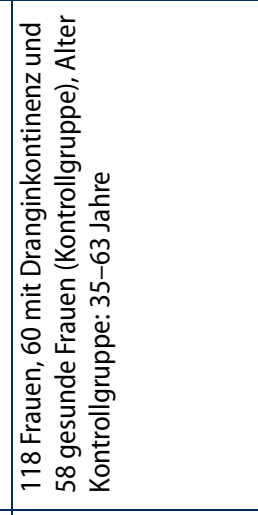 & 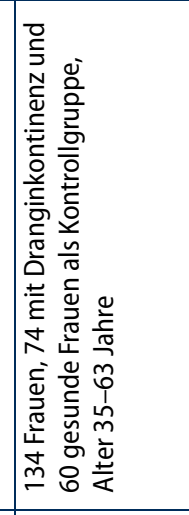 & 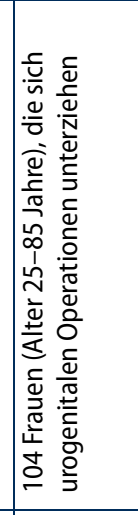 & 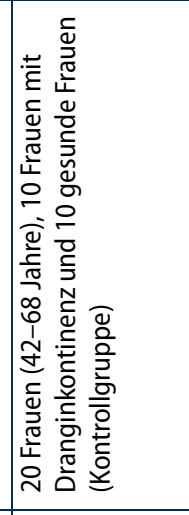 & 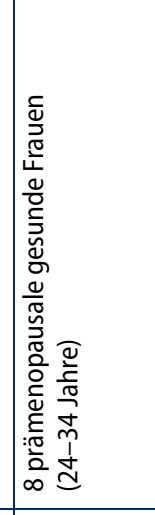 \\
\hline & & 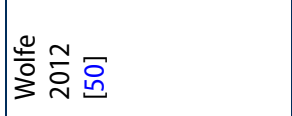 &  & 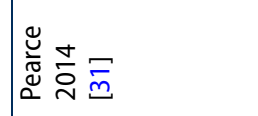 & & & 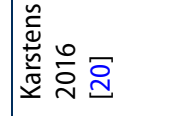 & 20 \\
\hline
\end{tabular}




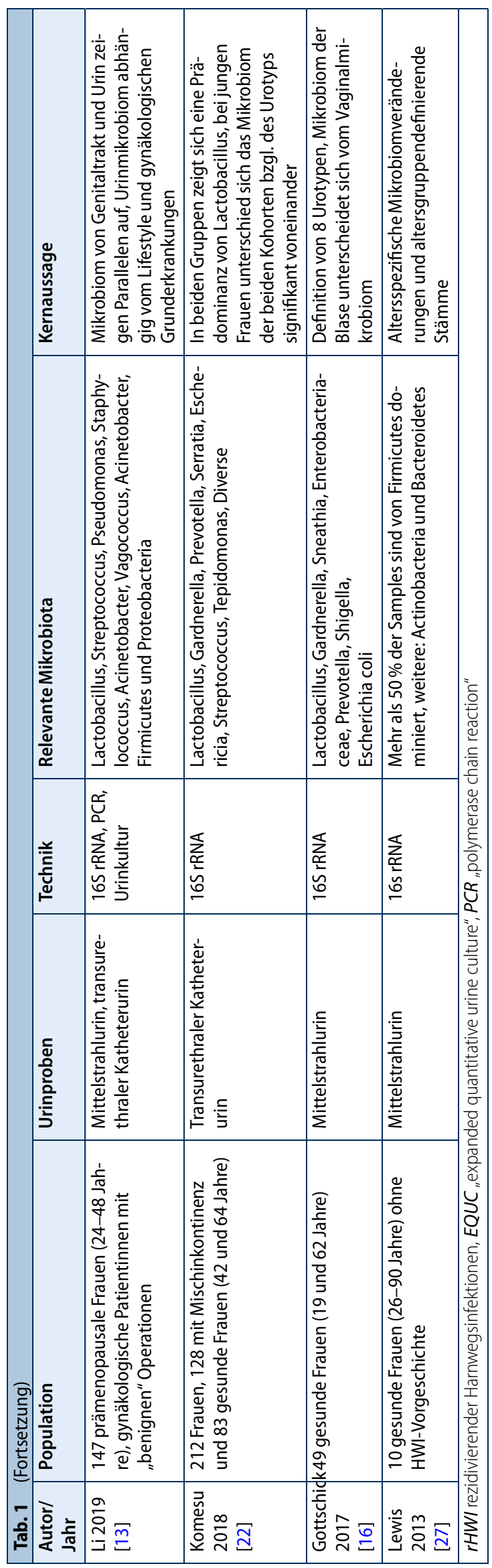

gezeigt. Vermehrtes Auftreten von Streptococcus und Staphylococcus spp. konnten bei Frauen mit ungeschütztem vaginalem und oralem Verkehr nachgewiesen werden [33]. Price et al. zeigten ebenfalls eine deutliche Variabilität der Blasenmikrobiota von 224 kontinenten Frauen. Die Mikrobiota wurden durch EQUC und NGS ("next-generation sequencing") untersucht. Dabei wurden sog. Urotypen bestimmt, definiert durch eine relative Abundanz eines Taxons von $>50 \%$. Der häufigste Urotyp war Lactobacillus (19\%), gefolgt von Streptococcus (9\%), Gardnerella (5\%) und Escherichia coli (4\%). Frauen, die einen Escherichia-coli-Urotyp vorwiesen, waren älter (mittleres Alter 60 Jahre, $p=0,005$ ) und postmenopausal $(p=0,008)$. Im Gegensatz dazu waren Frauen mit einem Gardnerella-Urotyp jünger (mittleres Alter 36 Jahre, $p<0,001$ ) hatten weniger Schwangerschaften hinter sich $(p<0,001)$ und waren übergewichtig (BMI 33,44, $p=0,04$; [34]). Das Blasenmikrobiom gesunder, asymptomatischer Frauen enthält mehrere Mikroorganismen, die als potenziell uropathogene Erreger bekannt sind ([16]; s. - Abb. 1).

\section{Blasenmikrobiomanalyse bei Frauen mit rHWl}

Lediglich eine Studiezur Untersuchung des Blasenmikrobioms von Frauen mit rHWI mittels 16S-RNA-Sequenzierung wurde identifiziert. Chen et al. untersuchten den Mittelstrahlurin von 39 postmenopausalen Frauen mit Dranginkontinenz und rHWl. Eingeschlossen wurden Patientinnen mit einer Anamnese rHWI und urodynamisch nachgewiesener Detrusorüberaktivität, refraktär zur konservativen Therapie und mehr als zwei verschiedenen Antimuskarinika über mindestens 12 Monate. Bei etwa $45 \%$ der Frauen konnte hier ein dominierender Erreger nachgewiesen werden (Escherichia coli, Enterococcus faecalis, Klebsiella pneumoniae oder Streptococcus spp.); $42 \%$ der Proben waren polymikrobiell unter anderem bestehend aus Morganella morganii, Klebsiella spp., Staphylococcus spp. und Enterococcus faecalis [14]. Hingegen zeigte sich eine verminderte bakterielle Diversität im Urin bei Frauen mit interstitieller Zystitis [40]. 
Tab. 2 Mikrobiom bei Harnwegsinfektionen

\begin{tabular}{|l|l|l|l|l|}
\hline Autor/Jahr & Population & Urinproben & Technik & Ergebnisse \\
\hline $\begin{array}{l}\text { Siddiqui } \\
2012 \text { [40] }\end{array}$ & $\begin{array}{l}\text { 8 Frauen mit der Diagnose } \\
\text { einer interstitiellen Zystitis } \\
\text { (27-67 Jahre) }\end{array}$ & Mittelstrahlurin & 16S rRNA & $\begin{array}{l}\text { Zusammensetzung, Verteilung und Diversität der vor- } \\
\text { kommenden Bakterien unterscheiden sich von gesunden } \\
\text { Frauen; Interstitielle Zystitis: 90\% Lactobacillus }\end{array}$ \\
\hline $\begin{array}{l}\text { Price 2016 } \\
\text { [32] }\end{array}$ & $\begin{array}{l}\text { 75 Frauen mit Symptomen von } \\
\text { HWl und 75 Kontrollpatientin- } \\
\text { nen aus der urogynäkologi- } \\
\text { schen Sprechstunde }\end{array}$ & $\begin{array}{l}\text { Katheterurin (trans- } \\
\text { urethral) }\end{array}$ & $\begin{array}{l}\text { EQUC und } \\
\text { Urinkultur }\end{array}$ & $\begin{array}{l}\text { Escherichia coli in der HWI-Kohorte, Streptococcus spp. und } \\
\text { Gardnerella vaginalis in Kontrollgruppe }\end{array}$ \\
\hline $\begin{array}{l}\text { Chen 2018 } \\
\text { [14] }\end{array}$ & $\begin{array}{l}\text { 39 postmenopausale Frauen mit } \\
\text { rez. HWl und therapierefraktärer } \\
\text { Dranginkontinenz (68-79 Jahre) }\end{array}$ & Mittelstrahlurin & $\begin{array}{l}\text { 16S rRNA } \\
\text { und Urin- } \\
\text { kultur }\end{array}$ & $\begin{array}{l}\text { Unter allen Probanden herrschen 5 Urotypen mit dominie- } \\
\text { rendem Keim in absteigender Häufigkeit: Corynebacteria- } \\
\text { ceae, Escherichia, Lactobacillus, Klebsiella, Staphylococcus } \\
\text { In den 9 schweren HWI-Fällen dominerte Escherichia coli } \\
\text { (versch. Stämme) }\end{array}$ \\
\hline $\begin{array}{l}\text { McDonald, } \\
\text { 2017 [29] }\end{array}$ & $\begin{array}{l}\text { 29 Frauen mit Symptomen einer } \\
\text { akuten Zystitis, 22 asymptoma- } \\
\text { tisch als Kontrollgruppe }\end{array}$ & Mittelstrahlurin & $\begin{array}{l}\text { 16S rRNA } \\
\text { und Urin- } \\
\text { kultur }\end{array}$ & $\begin{array}{l}\text { Diagnosestellung einer HWI anhand klinischer Kriterien } \\
\text { hat eine Fehlerrate von 33\%, v. a. bei komplizierten HWI } \\
\text { scheint ein Vergleich von Kultur und Sequenzierung sinn- } \\
\text { voll }\end{array}$ \\
\hline
\end{tabular}

Zudem wurde eine Studie identifiziert, welche EQUC bei Frauen mit rezidivierenden HWI nutzte. Price et al. fanden Unterschiede in der Diversität und Zusammensetzung des Bakterienspektrums bei 150 urogynäkologischen Patientinnen (50\% mit Symptomen einer HWI). 30\% der Frauen hatten eine vorhergehende antibiotische Therapie und $12 \%$ berichteten über Drangsymptomatik sowohl bei Frauen mit und ohne Symptome einer HWI. $36 \%$ der Frauen in der HWI-Gruppe und $17 \%$ der Frauen in der asymptomatischen Gruppe hatten eine vorhergehende urogynäkologische Operation. Streptococcus spp. und Gardnerella vaginalis wurden häufiger in der asymptomatischen, Escherichia coli in der symptomatischen Kohorte nachgewiesen [32]. Verglichen mit EQUC wurden bei der Urinkultur 67\% der Uropathogene der Gesamtkohorte und $50 \%$ bei Teilnehmern mit schweren Harnsymptomen übersehen. Eine größere pädiatrische Studie (570 Kinder im Alter von 1-4 Jahren) zeigte eine signifikant häufigere Wachstumspositivität bei EQUC als bei der Urinkultur. 20,6\% der mit EQUC nachgewiesenen signifikanten Isolate wurden mit der Urinkultur nicht detektiert [44].

\section{Therapeutischer Einsatz von Probiotika bei rHWI}

Insgesamt wurden 6 Studien zum Einfluss von Probiotika als Therapieoption von rHWI identifiziert (s. - Tab. 3). Hier- bei wurden verschiedene Protokolle und Dosierungen mit oraler oder vaginaler Applikation von Lactobacilluspräparaten, z. T. in Kombination mit Cranberry-Säften verwendet. Studien zur Anwendung von oralen Lactobacilluspräparaten lieferten widersprüchliche Erfolgsraten zur Senkung der Rezidivrate von rHWI. Eine doppelt verblindete randomisierte Studie mit $7 \mathrm{ge}$ sunden prämenopausalen Frauen zeigte, dass das Verhältnis von Lactobacillus spp. zu potenziell pathogenen Bakterien durch die Einnahme von Lactobacilluspräparaten nicht-signifikant verändert werden konnte [51]. Eine weitere randomisierte Studie konnte keine Reduktion der Rezidivrate bei 150 Frauen mit rHWI durch Escherichia coli unter Einnahme von Lactobacillusgetränken nachweisen [23]. Koradia et al. hingegen konnten in einer randomisierten Studie mit 81 prämenopausalen Frauen, bei der zwei orale Lactobacilluspräparate und ein Cranberry-Extrakt in Kombination verabreicht wurden, einen signifikanten Unterschied zur Placebogruppe in Bezug auf Häufigkeit und Dauer von rHWI zeigen [24]. Reid et al. fanden Hinweise dafür, dass orale Probiotika die vaginale Besiedlung mit potenziell pathogenen Bakterien reduzieren könne [36].

Therapeutischer Einsatz des fäkalen Mikrobiotatransfers bei rHWI

Der fäkale Mikrobiotatransfer (FMT) wird bisher in erster Linie zur Therapie von rekurrenten Clostridioides-difficile-Infektio- nen ( $r C D I)$ eingesetzt. Es gibt bisher 16 Fallberichte über den Effekt des FMT auf die Frequenz rHWI bei Frauen und Männern (s. - Tab. 4). Tariq et al. untersuchten im Rahmen einer retrospektiven Studie zum Einsatz des FMT bei rCDI Teilnehmer, die gleichzeitig unter rHWI litten. Hier zeigte sich im Vergleich zur Kontrollgruppe ein signifikant vermindertes Auftreten von HWl und ein Erregerprofil mit höherer Antibiotikaempfindlichkeit [43]. Auch in mehreren Fallberichten führte eine FMT-Behandlung bei Patienten mit rCDI oder Reizdarmsyndrom und gleichzeitigen $\mathrm{rHWI}$ zu einer deutlichen Reduktion oder einem Verschwinden dieser Infektionen [1, 19, 49]. Aira et al. konnten nach Anwendung von zwei FMT bei einer 93-jährigen Patientin mit rHWI und rCDI eine Symptomfreiheit über einen Beobachtungszeitraum von 299 Tagen und einen verminderten relativen Anteil von Enterobacterales von 74 auf $0,07 \%$ im Stuhlmikrobiom mit 16SrRNA-Sequenzierung nachweisen [1]. Drei weitere Fallberichte beschrieben den Einsatz des FMT bei nierentransplantierten $\mathrm{Pa}$ tientinnen $[6,17]$ bzw. einer dialysepflichtigen Frau vor geplanter Transplantation und rHWI [41]. In einem 12-oder 18-monatigen Nachbeobachtungsintervall wurden keine symptomatischen HWI mehr dokumentiert und eine Abnahme von potenziell pathogenen Bakterien in der Untersuchung des Urins festgestellt $[6,17,41]$. 
Tab. 3 Therapie mit Probiotika, randomisierte Kontrollstudie (RCT)

\begin{tabular}{|c|c|c|c|c|c|}
\hline Autor/Jahr & Population & Probiotikum & Urinproben & $\begin{array}{l}\text { Studiendesign } \\
\text { und Nachsorge }\end{array}$ & Ergebnisse \\
\hline $\begin{array}{l}\text { Wolff } 2019 \\
\text { [51] }\end{array}$ & $\begin{array}{l}7 \text { gesunde, prämeno- } \\
\text { pausale Frauen } \\
\text { (25-35 Jahre) }\end{array}$ & $\begin{array}{l}\text { Lactobacillus rhamnosus GR-1 und } \\
\text { Lactobacillus reuteri RC-14; oral als } \\
\text { Kapseln } 10^{9} \text { Organismen } 2 x \text { tgl. für } \\
40 \text { Tage }\end{array}$ & Urinkultur & $\begin{array}{l}\text { Doppelblinde } \\
\text { RCT; tägliche } \\
\text { Urinproben über } \\
3 \text { Monate }\end{array}$ & $\begin{array}{l}\text { Das Verhältnis von Lactobakterien } \\
\text { zu path. Keimen wird nicht durch } \\
\text { orale Probiotika verändert }\end{array}$ \\
\hline $\begin{array}{l}\text { Reid } 2003 \\
{[36]}\end{array}$ & $\begin{array}{l}64 \text { gesunde, präme- } \\
\text { nopausale Frauen } \\
\text { (19-46 Jahre) }\end{array}$ & $\begin{array}{l}\text { Lactobacillus rhamnosus GR- } 1 \text { und } \\
\text { L. fermentum RC- } 14 \text { als Kapseln } \\
10^{9} \text { Organismen } 1 x \text { tgl. Für } 60 \text { Tage }\end{array}$ & $\begin{array}{l}\text { Vaginalab- } \\
\text { striche }\end{array}$ & $\begin{array}{l}\mathrm{RCT} \text {, placebokon- } \\
\text { trolliert }\end{array}$ & $\begin{array}{l}\text { Orale Probiotika können die vagi- } \\
\text { nale Besiedlung mit pathogenen } \\
\text { Keimen vermindern }\end{array}$ \\
\hline $\begin{array}{l}\text { Reid } 1992 \\
\text { [35] }\end{array}$ & $\begin{array}{l}41 \text { erwachsene Frau- } \\
\text { en mit HWI }\end{array}$ & $\begin{array}{l}3 \text { Tage Antibiotikum (Cotrim oder } \\
\text { Norfloxacin), im Anschluss Lacto- } \\
\text { bacillus Supp. vaginal }\end{array}$ & Keine Angabe & Keine Angabe & $\begin{array}{l}\text { Eine lokale Lactobacillus Prophy- } \\
\text { laxe kann in Anschluss an eine } \\
\text { Antibiose die Rezidivwahrschein- } \\
\text { lichkeit vermindern }\end{array}$ \\
\hline $\begin{array}{l}\text { Kontiokari } \\
2001[23]\end{array}$ & $\begin{array}{l}150 \text { Frauen mit re- } \\
\text { zidivierenden HWls } \\
\text { durch E. coli }\end{array}$ & $\begin{array}{l}50 \mathrm{ml} \text { Cranberry-Saft tgl. für } 6 \mathrm{Mo}- \\
\text { nate oder } 100 \mathrm{ml} \text { Lactobacillus GG } \\
\text { drinks } 5 \text { x/Woche für } 1 \text { Jahr, oder } \\
\text { keine Intervention }\end{array}$ & Urinkultur & $\begin{array}{l}\text { RCT, } 12 \text { Monate } \\
\text { Nachsorge }\end{array}$ & $\begin{array}{l}\text { Cranberry-Saft kann wiederkeh- } \\
\text { rende HWI reduzieren, Lactobacil- } \\
\text { lus nicht }\end{array}$ \\
\hline $\begin{array}{l}\text { Koradia } \\
2019[24]\end{array}$ & $\begin{array}{l}81 \text { prämenopausale } \\
\text { Frauen (18-55 Jah- } \\
\text { re) mit }>2 \text { HWI im } \\
\text { letzten halben Jahr }\end{array}$ & $\begin{array}{l}\text { Cranberry } \\
\text { Extrakt + Lactob.acidophilus PXN } \\
35 \text { +Lactob. plantarum PXN } 47+ \\
\text { Vitamin A } 2 \text { x tgl. für } 26 \text { Wochen }\end{array}$ & Urinkultur & $\begin{array}{l}\mathrm{RCT} \text {, placebokon- } \\
\text { trolliert }\end{array}$ & $\begin{array}{l}\text { Die Häufigkeit und Dauer von HWI } \\
\text { konnte in der Interventionsgrup- } \\
\text { pe signifikant gesenkt werden }\end{array}$ \\
\hline
\end{tabular}

\section{Diskussion}

Wie die eingeschlossenen Studien zeigen, existiert ein Kernmikrobiom der Harnblase mit individueller Diversität (s. 0 Abb. 1 und - Tab. 1). In Abhängigkeit vom Alter, Vorerkrankungen, weiblichem Zyklus und sexueller Aktivität können Unterschiede in der Mikrobiotazusammensetzung festgestellt werden. Im Rahmen einer HWI findet eine Verschiebung zu Gunsten pathogener Mikroorganismen statt [32]. Außerdem nimmt die Diversität der Bakterienstämme ab [40].

Ein protektiver Effekt von Lactobacilli in Bezug auf symptomatische HWI konnte bisher nicht eindeutig nachgewiesen werden [38]. In einer Metaanalyse von 9 Studien mit insgesamt 735 Patienten mit rHWI und oraler oder vaginaler Applikation verschiedener Lactobacillusspezies fand sich keine signifikante Verringerung der Rezidivrate gegenüber einem Placebo [38]. Deshalb gab es bisher keine positive Bewertung in den Leitlinien $[3,8]$.

Die durchgeführte selektive Literatursuche ergab nur wenige Studien zur probiotischen Therapie von rHWI und zeigt ein erhebliches Forschungsdefizit auf. Die vorhandenen Studien haben Limitationen durch kleine heterogene Patientenkohorten, unterschiedliche Protokolle, Applikationswege und kurz Nachbeobachtungen. Vor allem fehlt der prä- und postinterven- tionelle Vergleich der Blasenmikrobiomzusammensetzung mit modernen Verfahren. Nur bestimmte zu definierende Urotypen würden von der Lactobacillustherapie profitieren. Es finden sich Hinweise, dass Lacotobacillen in der Harnblase protektiv im Hinblick auf das Auftreten von postoperativen HWI mit Enterobacterales und Pseudomonaden sein können [46]. Auch spielt das vaginale Milieu eine zentrale Rolle und bildet mit dem Darm als Ursprung auch fakultativ pathogener Erreger eine entscheidende Achse in der Pathogenese weiblicher HWI [2]. Ein bidirektionaler Austausch von Darm und urogenitalem Mikrobiom ist somit entscheidend zum Verständnis und der Prävention rHWI [30].

Auch die Therapiekosten spielen eine zunehmende Rolle. Gaitonde et al. führten eine ausführliche Kostenrechnung sowohl für die Diagnostik als auch für das Management von rHWI bei Frauen durch [15]. Die Kosten der akuten Behandlung mit Antibiotika reichen von $10 \$$ für orales Trimethoprim-Sulfamethoxazol (TMPSMX) bis hin zu etwa 4000 \$ für intravenöses (i.v.-)Ertapenem, je nach Medikamentenallergie und Resistenzlage. In der Studie wurden auch die Kosten für ein konservatives Management mit Probiotika, wie Lactobacillus, Cranberry-Tabletten oder Östrogentherapie berücksichtigt, die zwischen 50 und $1300 \$ /$ Jahr liegen [15].
Der FMT ist ein weiterer interessanter Therapieansatz bei rHWI. FMT wird in Kombination mit einer Antibiotikatherapie bereits erfolgreich als Therapie der Wahl bei rCDI eingesetzt [42]. Dieser Therapieansatz basiert auf einer Veränderung des Wirtmikrobioms. FMT wird mittels Koloskopie, Einläufen oder Kapseln mit aus Spenderstuhl gewonnenen Bakterien durchgeführt (s. Abb. 3). Stuhl- und Blutuntersuchen des Spenders im Rahmen eines umfangreichen Screenings minimieren das Risiko von Krankheitsübertragung. Die Annahme, dass uropathogene Bakterien, aus dem Gastrointestinaltrakt die Blase besiedeln, legt die Vermutung nahe, dass FMT nicht nur das intestinale Mikrobiom, sondern auch das Blasenmikrobiom verändern könnte.

Aktuell ist die rechtliche Lage des FMT aufgrund der COVID-19-Pandemie nicht sicher geklärt. Das Bundesinstitut für Arzneimittel und Medizinprodukte (BfArM) erließ am 01.04.2021 hierzu: „Die Behandlung mit FMT - im Rahmen von klinischen Studien oder als individueller Heilversuch sollte generell ausgesetzt werden" [5]. In der Urologie wäre die Anwendung potenziell ohne die aktuelle Einschränkung als individueller Heilversuch oder im Rahmen von AMG-Studien möglich. FMT allein oder in Kombination mit anderen probiotischen Therapien sollten auf ihre verlässliche und sichereWirksamkeit in der Behandlung von 


\begin{tabular}{|c|c|c|c|c|c|c|c|c|c|}
\hline 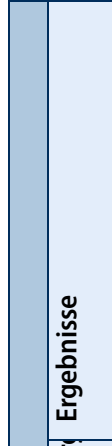 & 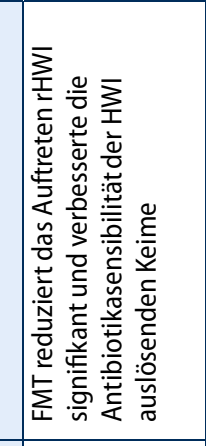 & 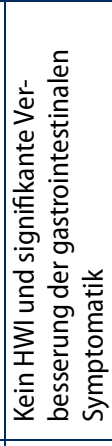 &  &  & 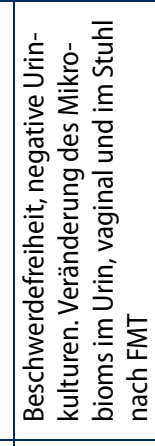 & 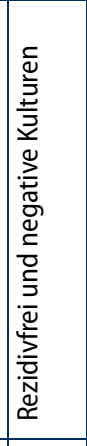 &  &  &  \\
\hline  & 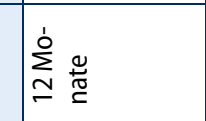 & 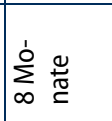 & 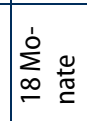 & 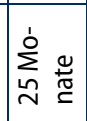 & 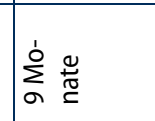 & 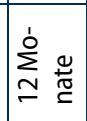 & 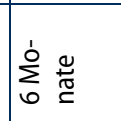 & 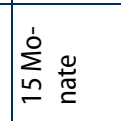 & 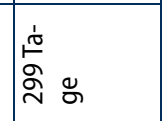 \\
\hline  & 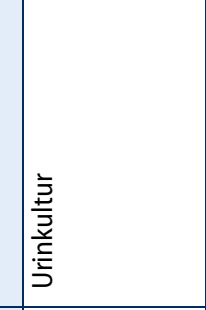 & 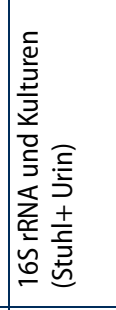 & 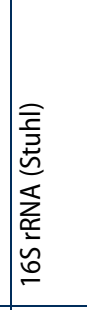 &  &  & 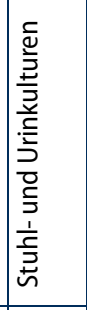 & 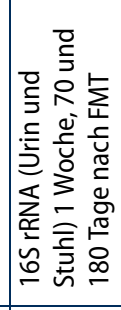 & 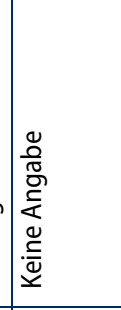 & 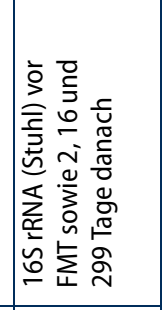 \\
\hline 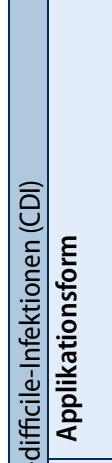 & 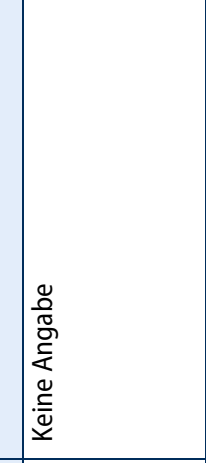 &  & 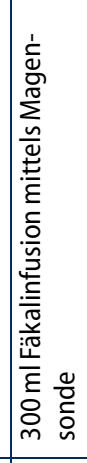 & & 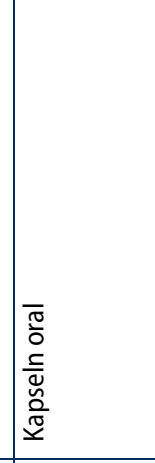 & 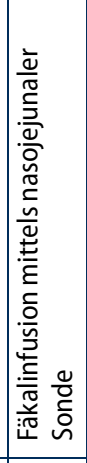 & 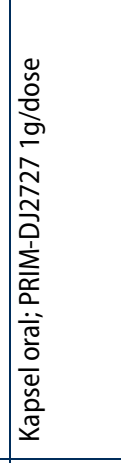 & 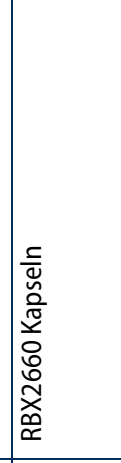 & 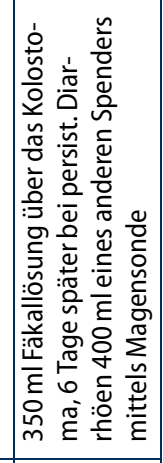 \\
\hline 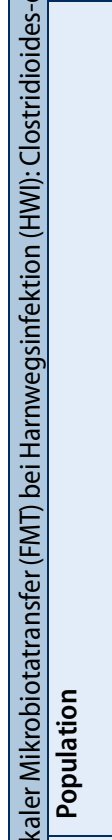 & 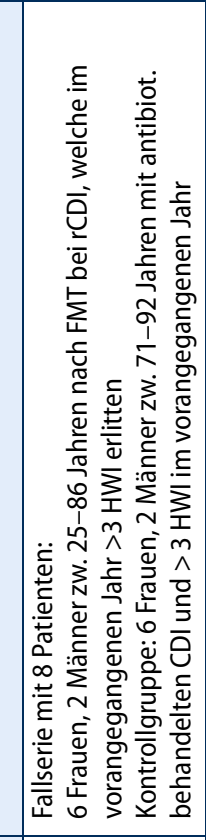 & 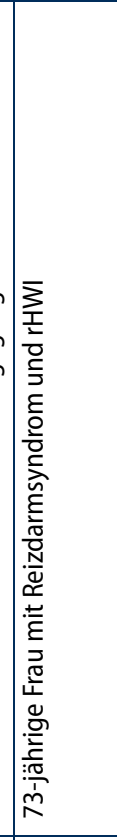 & 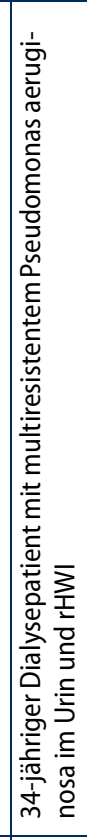 & 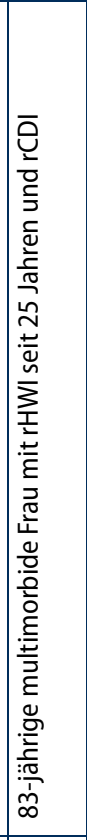 & 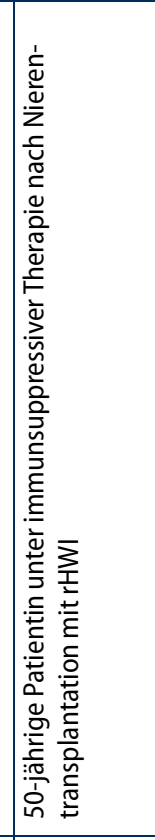 & 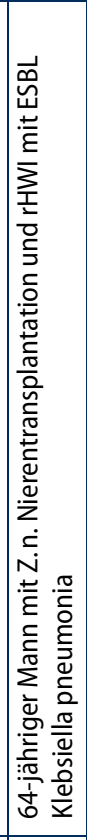 & 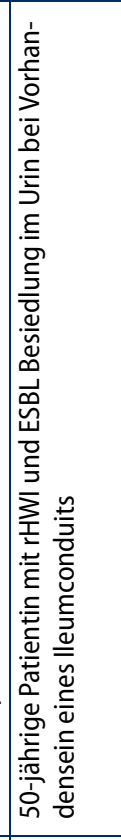 & 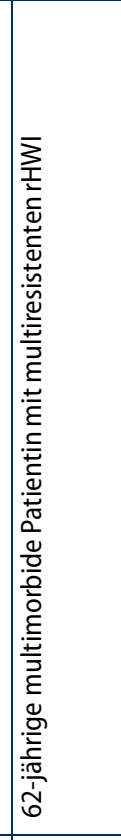 & 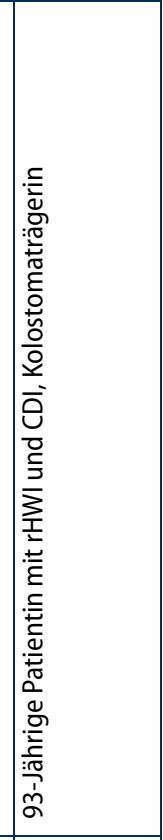 \\
\hline i⿱一兀) & 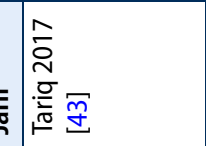 & 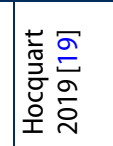 & 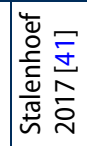 & $\Phi$ & $\begin{array}{l}\frac{\infty}{\bar{\sigma}} \\
\frac{\infty}{\bar{\sigma}} \\
\frac{\tilde{\omega}}{\infty} \\
\end{array}$ & 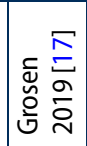 & 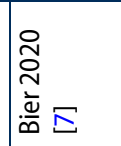 & 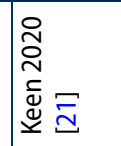 & 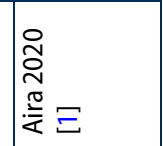 \\
\hline
\end{tabular}


Abb. $3<$ Das Vorgehen bei fäkaler Mirobiotatransplantation (FMT) sollte entsprechend der Vorgaben vom Bundesinstitut für Arzneimittel und Medizinprodukte (BfARM) und den verfügbaren Leitlinien entsprechen. (In Anlehnung an Stallmach [42])
Frauen mit rHWl in zentrenübergreifenden, randomisiert kontrollierten klinischen Studien überprüft werden, sobald die aktuellen COVID-19-bedingten Einschränkungen dieses zulassen.

Bakteriophagen, d.h. Viruspartikel, die auf Bakterien als Wirtszellen spezialisiert sind und diese lysieren können, bilden einen weiteren potenziellen Therapieansatz für rHWI. Theoretisch kann die Behandlung durch intravesikale Instillation mehrmals täglich erfolgen. Entdeckt im ersten Weltkrieg, wurden die Bakteriophagen zunächst v.a. in Sowjetunion zur Behandlung von Darmerkrankungen wie Cholera genutzt. Gegenwärtig ist die Bakteriophagentherapie in osteuropäischen Ländern wie Georgien, Armenien, der Ukraine und Russland ein zugelassenes Medikament. Aktuelle Präparationen aus Georgien und Russland bestehen üblicherweise aus einer Mischung virulenter Phagen, die sich in ihrer Wirtsspezifität überlappen und auch gegen antibiotikaresistente Bakterien aktiv sind [12].

In Deutschland liegt jedoch keine Zulassung vor und die Therapie kann ebenfalls nur als individueller Heilversuch durchgeführt werden. Allerdings liegen weltweit und über alle Indikationen hinweg bisher keine Ergebnisse aus interventionellen Studien vor, die die klinische Wirksamkeit von Phagen in der Therapie von HWI bestätigen würden.

Leitner et al. berichten z.B. über die Ergebnisse einer RCT, die Bakteriophagen zur Behandlung von HWI bei Patienten vor transurethraler Prostataresektion (TURP) in Georgien einsetzten. Die Verträglichkeit war gut, es konnte jedoch keine Überlegenheit in der Wirksamkeit im Vergleich zu systemisch verabreichten Antibiotika und einer Placeboblasenspülung gezeigt werden [26].

Der bisher ausgebliebende klinische Erfolg von Phagentherapien in Studien basiert auf verschiedenen Aspekten. Die Herstellung und Stabilität einer Phagenlösung ist schwierig und der Einsatz einer universell aktiven Phagenlösung weit entfernt. Zur spezifischen Therapie ist das Vorhalten von umfangreichen Phagensammlungen und das Erstellen eines individuellen sog. „Phagogramms“ im Voraus nötig, was sehr zeitaufwendig und teuer ist. Häufig können viele verschiedene Bakterienarten in einer kolonisierten oder infizierten Wunde vorhanden sein [25].Zukünftig sind sowohl die Entwicklung von Phagen im Rahmen der in Deutschland geltenden Regulationen, als auch Leitlinien für die Bewertung der Qualität, Sicherheit und Wirksamkeit von personalisierten Phagen Therapieansätzen erforderlich. Ein kombinierter Einsatz mit Antibiotika ist denkbar.

\section{Fazit für die Praxis}

- In der Harnblase lässt sich ein individuelles „Kernmikrobiom“ beschreiben, bei welchem unter anderem Lactobacilli eine wichtige protektive Rolle zu spielen scheinen.

- Das Blasenmikrobiom ist geschlechterabhängig und unterliegt vielen Einflüssen: Alter, Erkrankungen, antibiotische Therapien, Geschlechtsverkehr, hormoneller Status.

- Zu dem Mikrobiom einer "gesunden" Harnblase zählen auch potenziell pathogene Keime, wie Escherichia coli und Staphylococcus aureus. Eine vaginale Infektion oder Darmdysbiose kann ebenfalls zur Entwicklung einer symptomatischen Harnwegsinfektion führen.

- Moderne Methoden zur Urindiagnostik (EQUC [,expanded quantitative urine culture "] und NGS [,next-generation sequencing "]) sind aktuell nicht standardisiert und kostenintensiv. Ihr klinischer Nutzen ist strittig. 
- Ein weiterer potenzieller Therapieansatz ist der fäkale Mikrobiotatransfer, der im Rahmen von klinischen Studien geprüft werden sollte.

\section{Korrespondenzadresse}

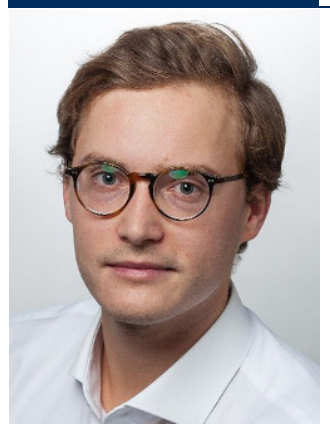

\section{T. Schiereck}

Urologische Klinik, Lukaskrankenhaus Neuss, Rheinland Klinikum

Preussenstr. 84, 41464 Neuss, Deutschland tim.schiereck@rheinlandklinikum.de

\section{Einhaltung ethischer Richtlinien}

Interessenkonflikt. T. Schiereck, S. Yeldan, J. Kranz, L. Schneidewind, F. Wagenlehner, I. Wieters, T. Otto und D. Barski geben an, dass kein Interessenkonflikt besteht.M.J.G.T.Vehreschild: Forschungsförderung: 3M, Astellas Pharma, Biontech, DaVolterra, Evonik, Gilead Sciences, Glycom, Immunic, MaaT Pharma, Merck/MSD, Organobalance, F. Hoffmann-La Roche Ltd, Seres Therapeutics, Takeda Pharmaceutical. Vorträge und Beratungstätigkeiten: Alb Fils Kliniken $\mathrm{GmbH}$, Arderypharm, Astellas Pharma, Basilea, BioMérieux, DaVolterra, Farmak International Holding $\mathrm{GmbH}$, Ferring, Gilead Sciences, Immunic AG, MaaT Pharma, Merck/MSD, Pfizer, F. Hoffmann-La Roche Ltd, Organobalance, SocraTec R\&D GmbH. Research grants: 3M, Astellas Pharma, Biontech, DaVolterra, Evonik, Gilead Sciences, Glycom, Immunic, MaaT Pharma, Merck/MSD, Organobalance, F. Hoffmann-La Roche Ltd, Seres Therapeutics, Takeda Pharmaceutical. Speaker fees and/or consulting: Alb Fils Kliniken $\mathrm{GmbH}$, Arderypharm, Astellas Pharma, Basilea, BioMérieux, DaVolterra, Farmak International Holding GmbH, Ferring, Gilead Sciences, Immunic AG, MaaT Pharma, Merck/MSD, Pfizer, F. Hoffmann-La Roche Ltd, Organobalance, SocraTec R\&D GmbH

Für diesen Beitrag wurden von den Autoren keine Studien an Menschen oder Tieren durchgeführt. Für die aufgeführten Studien gelten die jeweils dort angegebenen ethischen Richtlinien.

\section{Literatur}

1. Aira A, Rubio E, Vergara Gómez A et al (2020) rUTI resolution after FMT for Clostridioides difficile infection. Infect Dis Ther 10:1065-1071

2. Amabebe E, Anumba DOC (2020) Female gut and genital tract microbiota-induced crosstalk and differential effects of short-chain fatty acids on immune sequelae. Front Immunol 11:2184
3. AWMF S3 Leitlinien Harnwegsinfektionen (2017) Leitlinienprogramm DGU: Interdisziplinäre S3 Leitlinie: Epidemiologie, Diagnostik, Therapie, Prävention und Management unkomplizierter, bakterieller, ambulant erworbener Harnwegsinfektionen bei erwachsenen Patienten. Langversion 1.1-2. https://www.awmf.org/uploads/tx_ szleitlinien/043-044I_S3_Harnwegsinfektionen 2017-05.pdf. Zugegriffen: 30. Mai 2021 (AWMF Registernummer:043/044)

4. Bajic P, Wolfe AJ, Gupta GN (2019) The urinary microbiome. Urology 126:10-15

5. BfArM (2020) Maßnahmen zur Minimierung des Risikos einer möglichen Übertragung von SARSCoV-2 durch Fäkale Mikrobiota-Transplantation (FMT). https://www.bfarm.de/SharedDocs/ Risikoinformationen/Pharmakovigilanz/DE/RI/ 2020/RI-FMT.html.Zugegriffen: 12. Apr. 2021

6. Biehl LM, Cruz Aguilar R, Farowski F et al (2018) Fecal microbiota transplantation in a kidney transplant recipient with recurrent urinary tract infection. Infection 46(6):871-874

7. Bier N, Hanson B, Jiang Z-D et al (2020) 1694. Successful gut decolonization of extendedspectrum $\beta$-lactamase producing Klebsiella pneumoniae using oral lyophilized fecal microbiota transplant (FMT) in a woman with recurrent urinary tract infections. Open Forum Infect Dis 7(Supplement_1):S830-S831

8. Bonkat G, Bartoletti R, Bruyère $F$ et al (2020) Guidelines on urological infections. EAU, Arnhem

9. Brubaker L, Wolfe AJ (2015) The new world of the urinary microbiota in women. Am J Obstet Gynecol 213(5):644-649

10. Bschleipfer $T$ (2019) Mikrobiom, Harnwegsinfekt und $O A B$ - wie hängt das zusammen? UroForum 10(11):27-28

11. Caskurlu H, Culpan M, Erol B etal (2020) Changes in antimicrobial resistance of urinary tract infections in adult patients over a 5 -year period. Urol Int 104(3-4):287-292

12. Chanishvili N (2016) Bacteriophages as therapeutic and prophylactic means. Curr Drug Deliv 13(3):309-323

13. Chen C, Hao L, WeiWetal (2020) The female urinary microbiota in relation to the reproductive tract microbiota. Gigabyte. https://doi.org/10.46471/ gigabyte. 9

14. Chen Z, Phan M-D, Bates LJ et al (2018) The urinary microbiome in patients with refractory urge incontinence and recurrent urinary tract infection. Int Urogynecol J 29(12):1775-1782

15. Gaitonde S, Malik RD, Zimmern PE (2019) Financia burden of recurrent urinary tract infections in women. Urology 128:47-54

16. Gottschick C, Deng Z-L, Vital M et al (2017) The urinary microbiota of men and women and its changes in women during bacterial vaginosis and antibiotic treatment. Microbiome 5(1):99

17. Grosen AK, Povlsen JV, Lemming LE et al (2019) Faecal microbiota transplantation eradicated extended-spectrum beta-lactamase-producing Klebsiella pneumoniae from a renal transplant recipient with recurrent urinary tract infections. Case Rep Nephrol Dial 9(2):102-107

18. Hilt EE, McKinley K, Pearce MM et al (2014) Urine is not sterile. JClin Microbiol 52(3):871-876

19. Hocquart M, Pham T, Kuete Eet al (2019) Successful fecal microbiota transplantation in a patient suffering from irritable bowel syndrome and recurrent urinary tract infections. Open Forum Infect Dis 6(10):ofz398

20. Karstens L, Asquith M, Davin S et al (2016) Does the urinary microbiome play a role in urgency urinary incontinence and its severity? Front Cell Infect Microbiol 6:78

21. Keen EC, Tasoff P, Hink T et al (2020) Microbiome restoration by RBX2660 does not preclude recurrence of multidrug-resistant urinary tract infection following subsequent antibiotic exposure. Open Forum Infect Dis 7(3): ofaa42

22. Komesu YM, Richter HE, Carper B et al (2018) The urinary microbiome in women with mixed urinary incontinence compared to similarly aged controls. Int Urogynecol J 29(12):1785-1795

23. Kontiokari T, Sundqvist K, Nuutinen M et al (2001) Randomised trial of cranberry-lingonberry juice and Lactobacillus GG drink for the prevention of urinary tract infections in women. BMJ 322(7302):1571

24. Koradia P, Kapadia S, Trivedi Y et al (2019) Probiotic and cranberry supplementation for preventing recurrent uncomplicated urinary tract infections in premenopausal women. Expert Rev Anti Infect Ther 17(9):733-740

25. Kutter E, de Vos D, Gvasalia G et al (2010) Phage therapy in clinical practice. Curr Pharm Biotechnol 11(1):69-86

26. Leitner L, Ujmajuridze A, Chanishvili $\mathrm{N}$ et al (2021) Intravesical bacteriophages for treating urinary tract infections in patients undergoing transurethral resection of the prostate. Lancet Infect Dis 21(3):427-436

27. Lewis DA, Brown R, Williams J et al (2013) The human urinary microbiome; bacterial DNA in voided urine of asymptomatic adults. Front Cell Infect Microbiol 3:41

28. Magistro G, Marcon J, Eismann L et al (2020) Die Rolle des Mikrobioms in der Urologie. Urologe 59(12):1463-1471

29. McDonald M, Kameh D, Johnson ME et al (2017) A head-to-head comparative phase II study of standard urine culture and sensitivity versus DNA next-generation sequencing testing for urinary tract infections. Rev Urol 19(4):213-220

30. Meštrović T, Matijašić M, Perić M et al (2020) The role of gut, vaginal, and urinary microbiome in urinary tract infections. Diagnostics (Basel) 11(1):7

31. Pearce MM, Hilt EE, Rosenfeld AB et al (2014) The female urinary microbiome. mBio 5(4):e01283-14

32. Price TK, Dune T, Hilt EE et al (2016) The clinical urine culture. JClin Microbiol 54(5):1216-1222

33. Price TK, Wolff B, Halverson T et al (2020) Temporal dynamics of the adult female lower urinary tract microbiota. mBio 11(2):e00475-20

34. Price TK, Hilt EE, Thomas-White K et al (2020) The urobiome of continent adult women. BJOG 127(2):193-201

35. Reid G, Bruce AW, Taylor M (1992) Influence of three-day antimicrobial therapy and lactobacillus vaginal suppositories on recurrence of urinary tract infections. Clin Ther 14(1):11-16

36. Reid G, Charbonneau D, Erb J et al (2003) Oral use of Lactobacillus rhamnosus GR-1 and L. fermentum $\mathrm{RC}-14$ significantly alters vaginal flora. FEMS Microbiol Immunol 35(2):131-134

37. Reid G, Bruce AW (2006) Probiotics to prevent urinary tract infections. World J Urol 24(1):28-32

38. Schwenger EM, Tejani AM, Loewen PS (2015) Probiotics for preventing urinary tract infections in adults and children. Cochrane Database Syst Rev 12:CD8772

39. Siddiqui H, Nederbragt AJ, Lagesen Ket al (2011) Assessing diversity of the female urine microbiota by high throughput sequencing of $16 \mathrm{~S}$ rDNA amplicons. BMC Microbiol 11:244 
40. Siddiqui H, Lagesen K, Nederbragt AJ et al (2012) Alterations of microbiota in urine from women with interstitial cystitis. BMCMicrobiol 12:205

41. Stalenhoef JE, Terveer EM, Knetsch CW et al (2017) Fecal microbiota transfer for multidrug-resistant gram-negatives. Open Forum Infect Dis 4(2): ofx47

42. Stallmach A, Steube A, Grunert P et al (2020) Fecal microbiota transfer. Dtsch Arztebl Int 117(3):31-38

43. Tariq R, Pardi DS, Tosh PK et al (2017) Fecal microbiota transplantation for recurrent Clostridium difficile infection reduces recurrent urinary tract infection frequency. Clin Infect Dis 65(10):1745-1747

44. Thapaliya J, KhadkaP, Thapa Setal (2020) Enhanced quantitative urine culture technique, a slight modification, in detecting under-diagnosed pediatric urinary tract infection. BMC Res Notes 13(1):5

45. Thomas-White KJ, Hilt EE, Fok C et al (2016) Incontinence medication response relates to the female urinary microbiota. Int Urogynecol J 27(5):723-733

46. Thomas-White KJ, Gao X, Lin H et al (2018) Urinary microbes and postoperative urinary tract infection risk in urogynecologic surgical patients. Int Urogynecol J 29(12):1797-1805

47. Umek W (2019) Das urogenitale Mikrobiom und seine Bedeutung für den weiblichen Harntrakt. JUrol Urogynäkol AT 26(1):20-22

48. Vahlensieck W, Bauer HW, Piechota $H$ et al (2015) Rezidivierende Harnwegsinfektionen. Dtsch Arztebl 112(37):16

49. Wang T, Kraft CS, Woodworth MH et al (2018) Fecal microbiota transplant for refractory Clostridium difficile infection interrupts 25-year history of recurrent urinary tract infections. Open Forum Infect Dis 5(2): ofy16

50. Wolfe AJ, Toh E, Shibata N et al (2012) Evidence of uncultivated bacteria in the adult female bladder. JClin Microbiol 50(4):1376-1383

51. Wolff BJ, Price TK, Joyce CJ et al (2019) Oral probiotics and the female urinary microbiome. Int Urol Nephrol 51(12):2149-2159

\section{Urinary bladder microbiome analysis and probiotic treatment options for women with recurrent urinary tract infections}

Novel preventive measures and therapeutic approaches are needed to reduce the frequency of recurrent urinary tract infections (rUTI) and the associated emergence of multidrug-resistant uropathogens. The aim of this review is to systematically present the available evidence on the urinary bladder microbiome of healthy women and those with rUTIs. In addition, relevant studies on the efficacy of probiotics in rUTIs are presented in a structured manner. This will provide an overview on the current state of research and an outlook on treatment strategies beyond the usual antimicrobial options.

\section{Keywords}

Female bladder · Multidrug-resistant pathogens · 16S-rRNA-sequencing · Lactobacillus · Fecal microbiota transplantation 